Journal for ImmunoTherapy of Cancer

\title{
Nectin 4 is a novel TIGIT ligand which combines checkpoint inhibition and tumor specificity
}

\begin{abstract}
Adi Reches, ${ }^{1}$ Yael Ophir, ${ }^{1}$ Natan Stein, ${ }^{1}$ Inbal Kol, ${ }^{1}$ Batya Isaacson, ${ }^{1}$ Yoav Charpak Amikam, ${ }^{1}$ Afek Elnekave, ${ }^{1}$ Pinchas Tsukerman, ${ }^{1}$ Paola Kucan Brlic, ${ }^{2}$ Tihana Lenac, ${ }^{2}$ Barbara Seliger, ${ }^{3}$ Stipan Jonjic, ${ }^{2}$ Ofer Mandelboim (i) ${ }^{1}$
\end{abstract}

To cite: Reches A, Ophir Y, Stein N, et al. Nectin4 is a novel TIGIT ligand which combines checkpoint inhibition and tumor specificity. Journal for ImmunoTherapy of Cancer 2020;8:e000266. doi:10.1136/ jitc-2019-000266

- Additional material is published online only. To view please visit the journal online (http://dx.doi.org/10.1136/jitc2019-000266).

SJ and OM contributed equally.

Accepted 07 April 2020

Check for updates

(C) Author(s) (or their employer(s)) 2020. Re-use permitted under CC BY-NC. No commercial re-use. See rights and permissions. Published by BMJ.

${ }^{1}$ The Concern Foundation Laboratories at the Lautenberg Center for Immunology and Cancer Research, Hebrew University Hadassah Medical School, Jerusalem, Israel ${ }^{2}$ Department of Histology and Embryology, Faculty of Medicine, University of Rijeka, Rijeka,

Croatia

${ }^{3}$ Institute of Medical

Immunology, Martin-LutherUniversitat Halle-Wittenberg Halle, Sachsen-Anhalt, Germany

Correspondence to

Professor Ofer Mandelboim; oferm@ekmd.huji.ac.il

\section{ABSTRACT}

Background The use of checkpoint inhibitors has revolutionized cancer therapy. Unfortunately, these therapies often cause immune-related adverse effects, largely due to a lack of tumor specificity.

Methods We stained human natural killer cells using fusion proteins composed of the extracellular portion of various tumor markers fused to the Fc portion of human IgG1, and identified Nectin4 as a novel TIGIT ligand. Next, we generated a novel Nectin4 blocking antibody and demonstrated its efficacy as a checkpoint inhibitor in killing assays and in vivo.

Results We identify Nectin4 to be a novel ligand of TIGIT. We showed that, as opposed to all other known TIGIT ligands, which bind also additional receptors, Nectin4 interacts only with TIGIT. We show that the TIGIT-Nectin4 interaction inhibits natural killer cell activity, a critical part of the innate immune response. Finally, we developed blocking Nectin4 antibodies and demonstrated that they enhance tumor killing in vitro and in vivo.

Conclusion We discovered that Nectin4 is a novel ligand for TIGIT and demonstrated that specific antibodies against it enhance tumor cell killing in vitro and in vivo. Since Nectin4 is expressed almost exclusively on tumor cells, our Nectin4-blocking antibodies represent a combination of cancer specificity and immune checkpoint activity, which may prove more effective and safe for cancer immunotherapy.

\section{BACKGROUND}

Cancer immunotherapy with checkpoint inhibitors has shown great success in treating a wide variety of cancers. Given the immunomodulatory nature of these therapies, many of their side effects stem from immune overactivation. These are so common they have been named 'immune-related adverse events'. While many are easily managed, some of these autoimmune-like syndromes can prove fatal. ${ }^{1}$ To help avoid side effects, we searched for immunomodulatory molecules with specificity to tumors.

Natural killer (NK) cells are innate lymphocytes which are best known for their ability to kill virally infected and transformed cells. NK cell activity is regulated by a balance of signals they receive from a variety of activating and inhibitory receptors. ${ }^{2}$

The inhibitory receptor T-cell immunoreceptor with Ig and ITIM domains (TIGIT) is considered a major new target for cancer immunotherapy. ${ }^{3}$ It is expressed on most immune cells, ${ }^{3}{ }^{4}$ including NK cells and $\mathrm{CD}^{+}$tumor infiltrating lymphocytes (TILs) in non-small cell lung cancer, colon cancer and melanoma. ${ }^{5}$ Indeed, several anti-TIGIT immunotherapies have already reached phase I or II in clinical trials. ${ }^{5}$ TIGIT's known ligands arepoliovirus receptor (PVR), Nectin2 and Nectin3-some members of the Nectin family are overexpressed in many cancers. ${ }^{5-7}$ Some even serve as diagnostic indicators for several cancers. ${ }^{568}$ Interestingly, these ligands are also recognized by the coactivating receptors DNAM1 and CD96 (the latter of which is sometimes considered inhibitory) and by the inhibitory receptor CD112R. ${ }^{79}$

In contrast to the other Nectins, which are found extensively in adult tissues, ${ }^{10}$ Nectin4 is abundant during fetal development but its expression declines in adult life. Its expression, however, returns specifically in cancers of the breast, bladder, lung and pancreas, ${ }^{11}$ among others. Nectin 4 expression is also associated with poorer prognostic features. ${ }^{10}$

Here, we report that Nectin4 is a cancerspecific TIGIT ligand, and the only Nectin family member that interacts with TIGIT alone. We have developed a checkpoint blocking mAb against Nectin4, and show its efficacy in vitro and in vivo. Given the properties of Nectin4, our antibody represents a unique synergy between checkpoint inhibition and tumor specificity, which may prove beneficial in clinical use. 


\section{METHODS}

\section{Cells}

MDA-MB-453, SK-BR-3, T47D and lymph node carcinoma of the prostate (LNCAP) cells were maintained in Dulbecco's modified Eagle's medium and Raji cells were maintained in Roswell park memorial institute culture medium(RPMI) medium, both supplemented with $10 \%$ fetal calf serum, $1 \%$ Pen/Strep, $1 \%$ L-glutamine, $1 \%$ MEM Eagle and $1 \%$ sodium pyruvate. All cells were incubated in a humidified incubator with $5 \% \mathrm{CO}_{2}$ at $37^{\circ} \mathrm{C}$.

\section{Fusion proteins}

TIGIT-Ig, DNAM1-Ig, CD112-Ig, PVR-Ig, CD96-Ig, Nectin4-Ig ANPEP-Ig, PDGFRa-Ig, HAVcR-1-Ig, CD46Ig, EFNB2-Ig and murine TIGIT-Ig fusion proteins were cloned into an expression vector containing the mutated Fc portion of human IgG1 (Fc mut pIRESpuro3). The resulting constructs were transfected into HEK-293T cells using the TransIT-LT1 Transfection reagent (Mirus Bio). After 48 hours, transfected cells were subjected to antibiotic selection with $5 \mu \mathrm{g} / \mathrm{mL}$ puromycin (Sigma-Aldrich). Stable pools were analyzed for protein secretion by sodium dodecyl sulfate-polyacrylamide gel electrophoresis (SDS/PAGE). Supernatants were collected and purified on a HiTrap Protein G HP column in the High Pressure Perfusion Chromatography Station, BioCAD (PerSeptive Biosystems) as previously described. ${ }^{12}$ Nectin4-Ig was generated using these primers: Forward primer: GGGGAATT CGCCGCCA CCAT GCCCCTGT CCCTGGGA Reverse primer: GGGGGATCCGAGGGTGACAC TAGGTCC. HAVcR-Ig was generated using these primers: Forward primer: GGGAATTCGCCGCCACCATGCATC CTCAAGTGGTCA Reverse primer: GGGGATCCCCTT TAGTGGTATTGGCCGT. CD46-Ig was generated using these primers: Forward primer: GGGAATTCGCCGCCAC CATGGAGCCTCCCGGCCG Reverse primer: GGAG ATCTAAACTGTCAAGTATTCCTTCCTCA. EFNB2-Ig was generated using these primers: Forward primer: GGGCTAGC GCCGCCACCATG GCTG TGAGAAGG GACTCC Reverse primer: GGGGATCCGCAAATAA GGCCACTTCGGAAC. ANPEP-Ig was generated using these primers: Forward primer: AAAACTAGTTACTCCC AGGAGAAGAACAAGA Reverse primer: TTTCTCGA GCTATTTGCTGTTTTCTGTGAACC. PDGFRa-Ig was generated using these primers: Forward primer: AAAG CTAGCGCCGCCACCATGGGGACTTCCCATC Reverse primer: TTTGGATCCGCCACCGTGAGTTC. DNAM1-Ig was generated using these primers: Forward primer: CCGATATCGCCGCCACCATGGATTATCCTACTTTAC TTTTG Reverse primer: CGGATCCACAAAGAGG GTATATTGGTTAT. TIGIT-Ig was generated using these primers: Forward primer: ATGCGCTGGTGTCTCCTCCT Reverse primer: TCTTCACAGAGACTGGTTAG. Murine TIGIT-Ig was generated using these primers: Forward primer: CCGAATTCGCCGCCACCATGCATGGCTG GCTGCTCCT. Reverse primer: CGGATCCGGGGCAGTC TGGAACTGAG. CD112R-Ig was generated using these primers: Forward primer: ATGCAGATCCCACAGGCGCC Reverse primer: CCCCCATTCTGCGGGCAGAC.

The fusion proteins used in this work were regularly assayed by SDS protein gels to ensure that the proteins are not degraded. Protein purity of all Ig fusion proteins used approached $100 \%$.

\section{Generation of lentivirus overexpression}

Nectin 4 was cloned into the pHAGE-DsRED(-)-eGFP $(+)$ lentiviral vector which also contains GFP. Lentiviruses were generated in $293 \mathrm{~T}$ cells using a transient threeplasmid transfection protocol as previously described. ${ }^{13}$ Transduction efficiency into Raji cells was assessed by GFP expression and only cell populations with $>90 \%$ efficiency were used for experiments. Primers sequences: Human Nectin4 forward: GGGCGGCCGCGCCGCCACCATGCC CCTGTCCCTGGGA, Human Nectin4 reverse: GGAC CGGTTCAGACCAGGTGTCCCCGC. Murine Nectin4 forward: GCGGCGGCCGCGCCGCCACCAGCCAATG CCCCTGT, Murine Nectin4 reverse: CGCACCGGTGGC CTGGGTCAGACC.

\section{Mouse thymoma (BW) assay}

The generation of chimeric TIGIT and DNAM1 and expression in BW cells was previously described in details. ${ }^{14}{ }^{15} \mathrm{In}$ brief, we expressed chimeric proteins composed of the extracellular portions of TIGIT and DNAM1 fused to the mouse $\zeta$-chain in the BW murine thymoma cell line. The BW assay was performed as previously described. Briefly, BW or BW transfected cells were incubated with irradiated targets $(6000 \mathrm{rad})$ at 1:1 effector to target ratio. After 48 hours, the supernatants were collected and the level of interleukin-2 (IL2) was quantified by sandwich ELISA using anti IL2 mAbs (BioLegend).

\section{Generation of anti-Nectin4 antibodies}

The anti-Nectin $4 \mathrm{mAbs}$ (clone .01 and clone .05) were generated by immunizing mice with Nectin4-Ig fusion proteins and then by using standard hybridoma techniques. The hybridomas were cultured in RPMI-medium and the antibodies were purified on a protein G column. All antibodies generated are of the IgG1 isotype.

\section{Flow cytometry}

Extracellular fluorescence-activated cell sorting (FACS) staining for Nectin4 (MAB2659, R\&D Systems), Nectin2 (BLG-337402, BioLegend), PVR (MAB25301, R\&D Systems) DNAM1 (BLG-338302, BioLegend) and TIGIT (MAB7898, R\&D Systems) was performed with $0.2 \mu \mathrm{g}$ antibody per 100000 cells. Binding was detected by a secondary antibody (Alexa Flour 647 anti-mouse IgG, Jackson, cat. 115-606-062), at a dilution of 1:200, incubated for 1 hour on ice. Extracellular FACS staining with TIGIT-Ig, DNAM1-Ig, CD112-Ig, CD96-Ig, PVRIg, Nectin4-Ig and murine TIGIT-Ig fusion proteins was performed with $3 \mu \mathrm{g} /$ well. Binding was detected by a secondary antibody (Allophycocyanin antihuman IgG, Jackson, cat. 709-136-098), at a dilution of 1:200, incubated for 1 hour on ice. For antibody blocking 

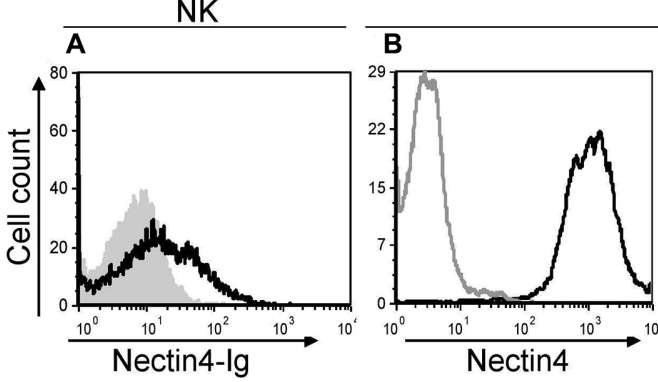

Raji
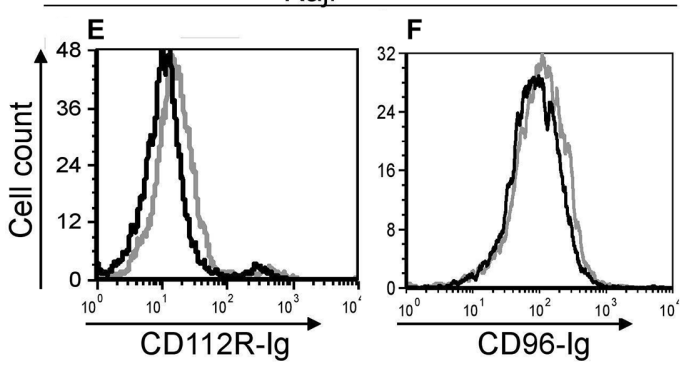

Raji
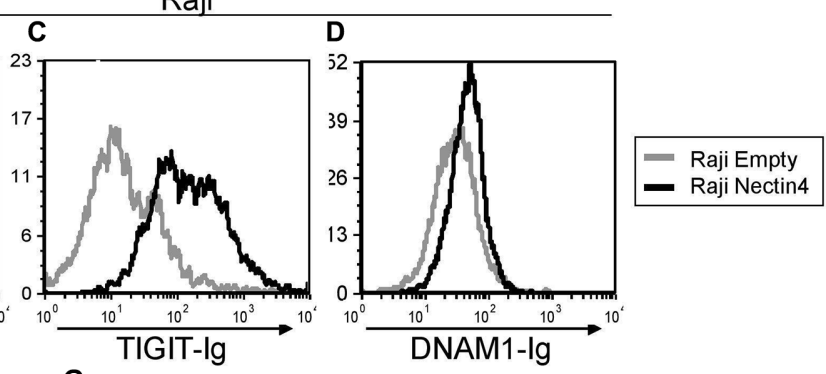

$\mathbf{G}$

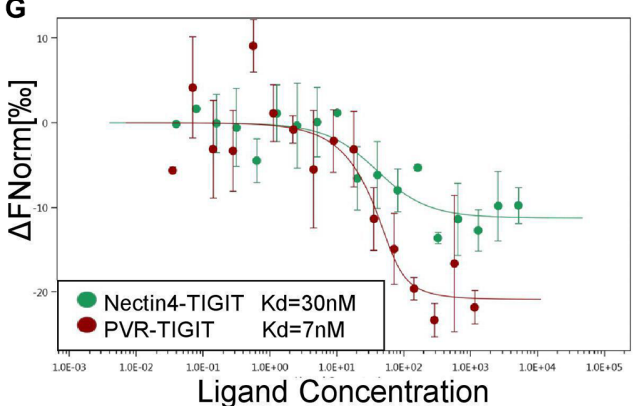

Figure 1 TIGIT but not DNAM1, CD112R or CD96-interacts with Nectin4. (A) FACS staining of IL-2 activated primary NK cells with Nectin4-lg. Gray filled histogram represents background staining with secondary antibody only; black line histogram represents specific binding as indicated. (B-F) FACS staining of Raji cells transfected either with an empty vector as control (gray histograms) or with Nectin4 (black histograms). Cells were stained with commercial anti-Nectin4 mAb (B), TIGIT-Ig (C), DNAM1-lg (D), CD112-lg (E) or CD96-lg (F). Figures show one representative experiment out of three performed. Graph depicting the mean fluorescence intensity values of the stainings appears in online supplementary figure 2. (G) Direct binding of Nectin4 to TIGIT. The binding of fluorophore-labeled TIGIT-Ig and its ligands PVR-Ig (red) and Nectin4-Ig (green) was determined using MST. Measurements were repeated with at least three independent protein preparations. FACS, fluorescence-activated cell sorting; IL-2, interleukin-2; MST, microscale thermophoresis; NK, natural killer.

experiments, cells were preincubated with the indicated antibody for 1 hour on ice. All steps were performed in a Phosphate buffered saline (PBS)-based buffer containing $5 \%$ bovine serum Albumin (BSA) and $0.05 \%$ sodium azide. All staining was assessed using FACS with CellQuest software and analyzed with FCSexpress.

\section{Microscale thermophoresis}

The microscale thermophoresis (MST) experiments were performed as previously described. ${ }^{16}$ Briefly, labeled TIGIT-Ig was mixed with Nectin4-Ig or PVR-Ig (figure 1G) and labeled Nectin4-Ig was mixed with either anti-Nectin4 mAb clone .01 or clone 05 (figure $2 \mathrm{E}$ ) in standard capillaries at $25^{\circ} \mathrm{C}$. Titration of the non-fluorescent antibodies resulted in a gradual change in thermophoresis, which was plotted as a change in the normalized fluorescence (Fnorm), to yield a binding curve.

\section{NK cell killing assay}

To evaluate NK cell cytotoxic activity against target cells, $\left[{ }^{35} \mathrm{~S}\right]$ release assays were performed as described previously. ${ }^{17}$ For antibody blocking, cells were preincubated with the indicated antibodies for 1 hour on ice. The reaction mixtures were incubated for 5 hours. Percentages of killing were calculated as follows: [counts per minute (cpm) (sample) - cpm (spontaneous release) $] /[\mathrm{cpm}$ (total release) - cpm (spontaneous release) $] \times 100$.

\section{Western blot analysis}

Lysates of the cells were prepared and SDS gel electrophoresis was performed. Proteins were transferred onto a nitrocellulose membrane with the tank blot procedure and specific protein bands were detected using antibodies detecting murine Nectin4 (MAB3116, R\&D Systems (1:200)) or Glyceraldehyde 3-phosphate dehydrogenase (GAPDH) (SC-32233, Santa Cruz (1:1000)) as loading control. Antibodies were diluted in 5\% BSA in PBS. Chemiluminescence from detection antibodylinked horseradish peroxidase (Jackson ImmunoResearch, Pennsylvania, USA) was detected.

\section{Mouse tumor development}

All experiments were performed using 6-8weeks severe combined immunodeficiency disease (SCID)-beige female mice. All mice were housed under Specific pathogen-free (SPF) conditions, in normal light/dark cycles at $22^{\circ} \mathrm{C} \pm 2^{\circ} \mathrm{C}$. All experiments were performed in accordance with the guidelines of the ethics committee of the Hebrew University Medical School (Ein-Kerem, Jerusalem). Every group of mice contained seven females $(n=7)$. Xenografts were generated by administering the indicated cells subcutaneously into the left flank region. Injections of $75 \mathrm{ug}$ anti-Nectin 4 clone .05 or control (antiCD3, InVivoMAb-clone 17A2) antibodies were administrated intraperitoneally twice a week, starting from the time point when the tumor could be seen as a white dot 

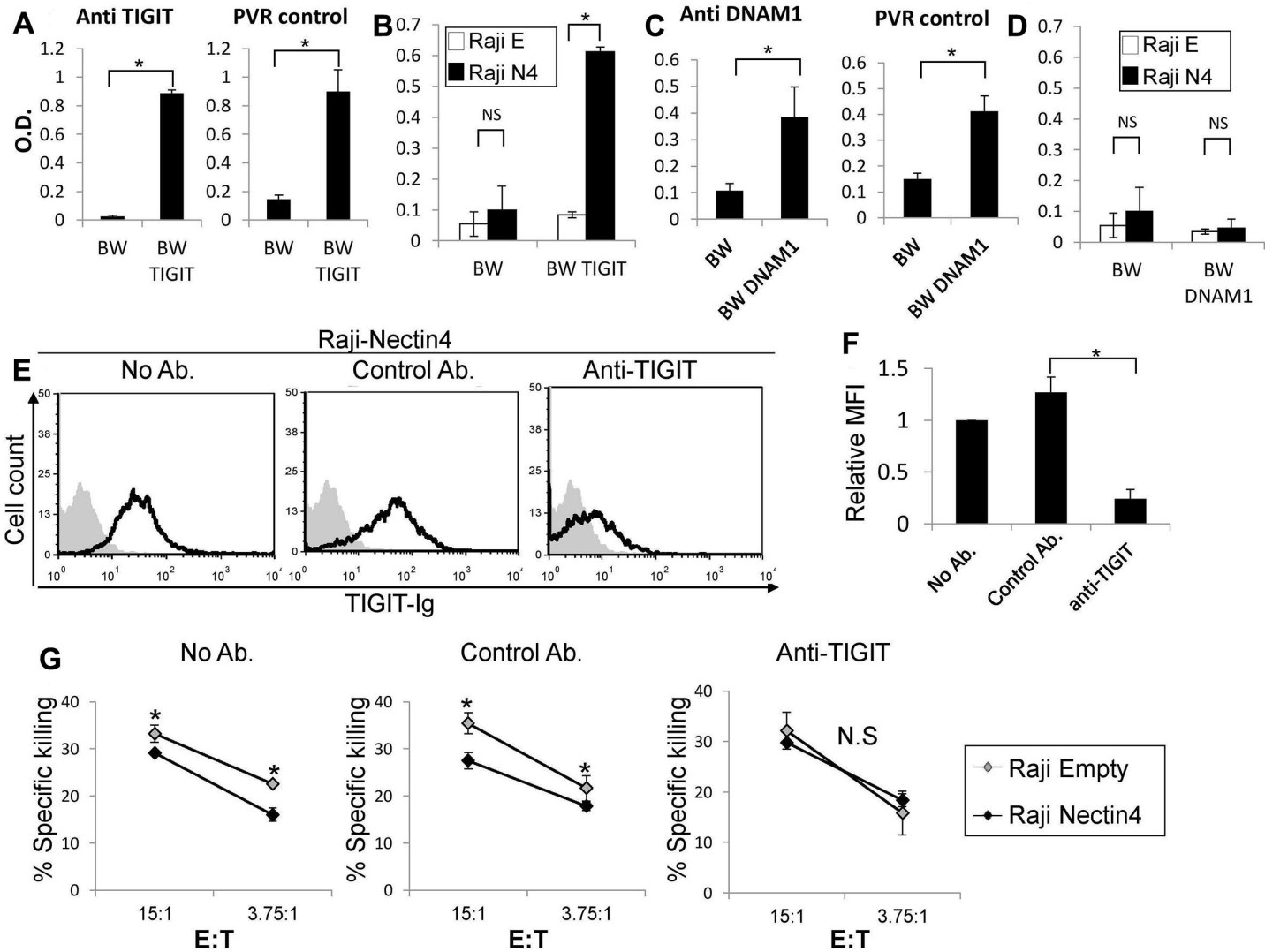

Figure 2 .Nectin4 inhibits NK cytotoxicity via TIGIT. (A, C) IL2 secretion by parental BW (A, C), (A) BW-TIGIT and (C) BW-DNAM1 cells. IL2 secretion was determined by ELISA $(\mathrm{od} 650 \mathrm{~nm}$ ) following incubation with control anti-TIGIT or antiDNAM1 antibodies (left in a and C) or with PVR expressing cells (right in a and C). (B, D) IL2 secretion of parental BW (B, D), (B) BW-TIGIT and (D) BW-DNAM1 cells. IL2 secretion was determined by ELISA (od $650 \mathrm{~nm}$ ) following incubation with Raji cells transfected either with an empty vector (Raji E) as a control, or with Nectin4 (Raji N4). Figure shows one representative experiment out of 3 performed. ${ }^{*} \mathrm{P}<0.05$. (E) FACS staining of Raji cells overexpressing Nectin 4 with TIGIT-Ig. TIGIT-Ig was preincubated with no antibody (left), with a control mAb (anti-CD99 mAb clone 12E7, middle) or with anti-TIGIT blocking antibody (mAb \#4 generated as described previously, ${ }^{14}$ right). Black line histograms represent TIGIT-Ig binding. Gray filled histograms represent background staining of the secondary antibody only. (F) Mean fluorescence intensity (MFI) values of the TIGIT-Ig staining shown in (E) relative to NO antibody staining, ${ }^{*} \mathrm{p}<2 \times 10^{-4}$. (G) $\left[{ }^{35} \mathrm{~S}\right]$ methionine-labeled Raji cells transfected either with an empty vector as control (Raji empty - gray) or with Nectin4 (Raji Nectin4-black), were incubated for 5 hours with NK cells. NK cells were preincubated with no antibody (left), with a control antibody (anti-CD99 mAb clone 12E7, middle) or with an anti-TIGIT antibody (mAb \#4 generated as described previously, ${ }^{14}$ right). The effector to target $(\mathrm{E}: \mathrm{T})$ ratios are indicated on the $\mathrm{x}$-axis. Figure shows one representative experiment out of three performed. Shown is the relative average killing $\pm S D$, ${ }^{*} \mathrm{p}<0.05$. FACS, fluorescence-activated cell sorting; IL2, interleukin-2; NK, natural killer; NS, not significant.

under the skin. The mice were monitored daily, and sacrificed at any indication of illness such as bristled fur, difficult breathing, or tremor, among others. When tumors reached a maximal volume of $1000 \mathrm{~mm}^{3}$, all mice were sacrificed. No differences were observed between the various groups in their general health at baseline.

\section{Statistical analysis}

All p values were determined using Student's t-test, except the Kaplan-Meier curves $p$ values, which were generated from the $\mathrm{X}^{2}$ score with $1 \mathrm{df}$.

\section{RESULTS}

Nectin4 interacts with the immune checkpoint receptor TIGIT

In testing whether certain tumor markers interact with NK cells, we generated fusion proteins composed of the extracellular portion of various tumor markers, including Nectin4, fused to the Fc portion of Human IgG1 (Nectin4-Ig). We next stained human NK cells and observed staining only with Nectin4-Ig (figure 1A, online supplementary figure 1a-e). Since other Nectin family members interact with TIGIT, DNAM1, CD112R and CD96, ${ }^{7}$ we next tested whether Nectin4 interacts with these receptors. We overexpressed Nectin 4 on Raji cells, which do not normally express any ligands of these receptors, including Nectin4 (figure 1B, online supplementary figure $1 \mathrm{f}-\mathrm{h}$ ). We stained the transfected cells with TIGIT-Ig, DNAM1-Ig, CD112R-Ig and CD96-Ig. Interestingly, Nectin4 overexpression led to increased binding of the inhibitory receptor TIGIT-Ig alone (figure 1C-F), uniquely allowing for a purely inhibitory effect.

To demonstrate that Nectin4 interacts directly with TIGIT, we used MST. We found that TIGIT binds Nectin4 
with relatively high affinity, similar to that of PVR-TIGIT binding (figure 1G). Collectively, we concluded that Nectin 4 is not only a TIGIT ligand, but also the only Nectin family member that interacts exclusively with TIGIT, and not with DNAM1, CD96 or CD112R.

\section{Nectin4 is a functional ligand for TIGIT}

To further determine the binding of Nectin4 to TIGIT, we used a cell-based reporter assay. We expressed a chimeric protein composed of TIGIT fused to mouse $\zeta$-chain in the mouse BW thymoma cell line and named the cells BW TIGIT (online supplementary figure 1i). In this system, BW cells secrete IL2 on engagement of the chimeric TIGIT receptor by its ligand (present on target cells), thus reporting for binding. We determine the amount of IL2 in the supernatants using ELISA. To test that the reporter system is functional, we initially triggered the chimeric TIGIT protein with antibody or incubated the BW TIGIT cells with cells expressing PVR, a known TIGIT ligand. In both cases, IL2 secretion was observed when BW TIGIT cells were used, while little or no IL2 secretion was detected when parental BW cells were used (figure 2A). We then incubated BW parental and BW TIGIT cells with RAJI cells transfected either with Nectin4 (RAJI N4) or empty vector (RAJI E). Little or no IL2 was observed when the parental BW cells were incubated with RAJI cells with or without Nectin4 expression (figure 2B). In contrast, when BW TIGIT cells were incubated with RAJI Nectin 4 cells, significantly more IL2 was secreted as compared with RAJI Empty cells (figure 2B), indicating, using an isolated reporter system, that TIGIT interacts with Nectin4.

As a negative control, we used DNAM1 that is also known to interact with PVR. ${ }^{5}$ BW expressing DNAM1 cells were generated, in a similar manner to the BW TIGIT cells described above, and expression of DNAM1 was verified (online supplementary fiugre $1 \mathrm{j}$ ). We verified that the BW DNAM1 reporter cells function by stimulating with antiDNAM1 mAb or with cells expressing PVR (figure 2C). In agreement with the lack of Nectin 4 recognition by DNAM1-Ig, no significant IL2 expression was observed on BW DNAM1 incubation with either Raji Empty vector or Raji Nectin4 cells (figure 2D).

We next sought to address the impact of TIGITNectin4 interaction using primary human NK cells. We first tested whether a blocking anti-TIGIT $\mathrm{mAb}^{14}$ impairs this interaction. On preincubation of the anti-TIGIT mAb with TIGIT-Ig, binding of TIGIT-Ig to cells overexpressing Nectin4 was reduced (figure 2E, summarized in figure $2 \mathrm{~F}$ ). We also performed NK cytotoxicity assays with activated primary human NK cells that express TIGIT (online supplementary figure $1 \mathrm{k}$ ). As expected, expression of Nectin 4 on Raji cells led to reduced NK cell cytotoxicity (figure 2G; see No Ab and Control Ab). Preincubation of NK cells with the blocking anti-TIGIT $\mathrm{mAb}$ abrogated this difference (figure 2G). These results demonstrate that Nectin4 is indeed a functional ligand for TIGIT.

\section{Generation and characterization of Nectin4 blocking} antibodies

Nectin 4 is a well-known tumor marker, as it is overexpressed on various tumors including breast, bladder, lung and pancreas. ${ }^{11}$ Therefore, we wanted to generate checkpoint blocking antibodies against Nectin4. We immunized mice against Nectin4-Ig in order to generate hybridoma cell lines and select those that secrete blocking antibodies. Two of them, clones .01 and .05 , are shown in figures 2 and 3 . First, we confirmed specific binding of these antibodies to Nectin 4 by staining Raji cells overexpressing Nectin4 (figure 3A,B). We then assessed the ability of these antibodies to block TIGIT-Ig binding (figure 3C,D). Both antibodies effectively block Nectin4TIGIT interaction. Using MST, we determined the affinities of the mAbs to Nectin4, which are in the $\mathrm{pM}$ range (figure $3 \mathrm{E}$ ). To evaluate functional ability of the antiNectin 4 mAbs to block TIGIT-mediated inhibition, we used Raji cells overexpressing Nectin4. Preincubation of target cells with either antibody led to increased killing by NK cells (figure $4 \mathrm{~A}$ ).

To establish that endogenous Nectin 4 is a central ligand for TIGIT, even in the context of other TIGIT ligands, we assayed various cell lines (breast cancer cell lines MDAMB-453, SK-BR-3, T47D and prostate cancer LNCAP) that naturally express Nectin4 (figure 4B-E) along with other TIGIT ligands. We performed NK cytotoxicity assays on these cells as well. Preincubation of all cell lines with either anti-Nectin 4 antibody led to increased killing by primary NK cells (figure 4F-I). Accordingly, we concluded that Nectin4 is a new immune checkpoint ligand, and that our antibodies can functionally block its inhibitory effect.

\section{Anti-Nectin4 antibody can attenuate tumor growth in vivo}

Before moving to in vivo models, we first investigated whether murine Nectin4 interacts with murine TIGIT. We overexpressed murine Nectin4 in Raji cells (figure 5A) and stained these cells with the murine TIGIT-Ig. As no binding was detected (figure 5B), we concluded that the orthologous system in mice is irrelevant.

Therefore, to demonstrate the functionality of the interaction of Nectin4 and TIGIT in vivo, SCID-beige mice were subcutaneously implanted with Raji cells with either human Nectin4 overexpression or empty vector, with or without the addition of human NK cells. In the absence of NK cells, Nectin4 overexpression did not lead to any detectable difference in tumor size and weight (figure 6A,B). In contrast, when NK cells were included, tumor size was significantly larger in Raji tumors overexpressing Nectin4 (figure 6C,D). Therefore, we show that Nectin4 is an immune checkpoint ligand in vivo.

In order to test the efficacy of our antibodies in vivo, we used them along with human NK cells to treat mice with Raji tumors overexpressing Nectin4. We compared tumor growth with injection of either a control antibody or anti-Nectin 4 clone $.05 \mathrm{mAb}$ (selected due to greater blocking as shown in figure 2C,D). We found that clone .05 successfully suppresses tumor growth (figure 6E,F). 


\section{Raji}
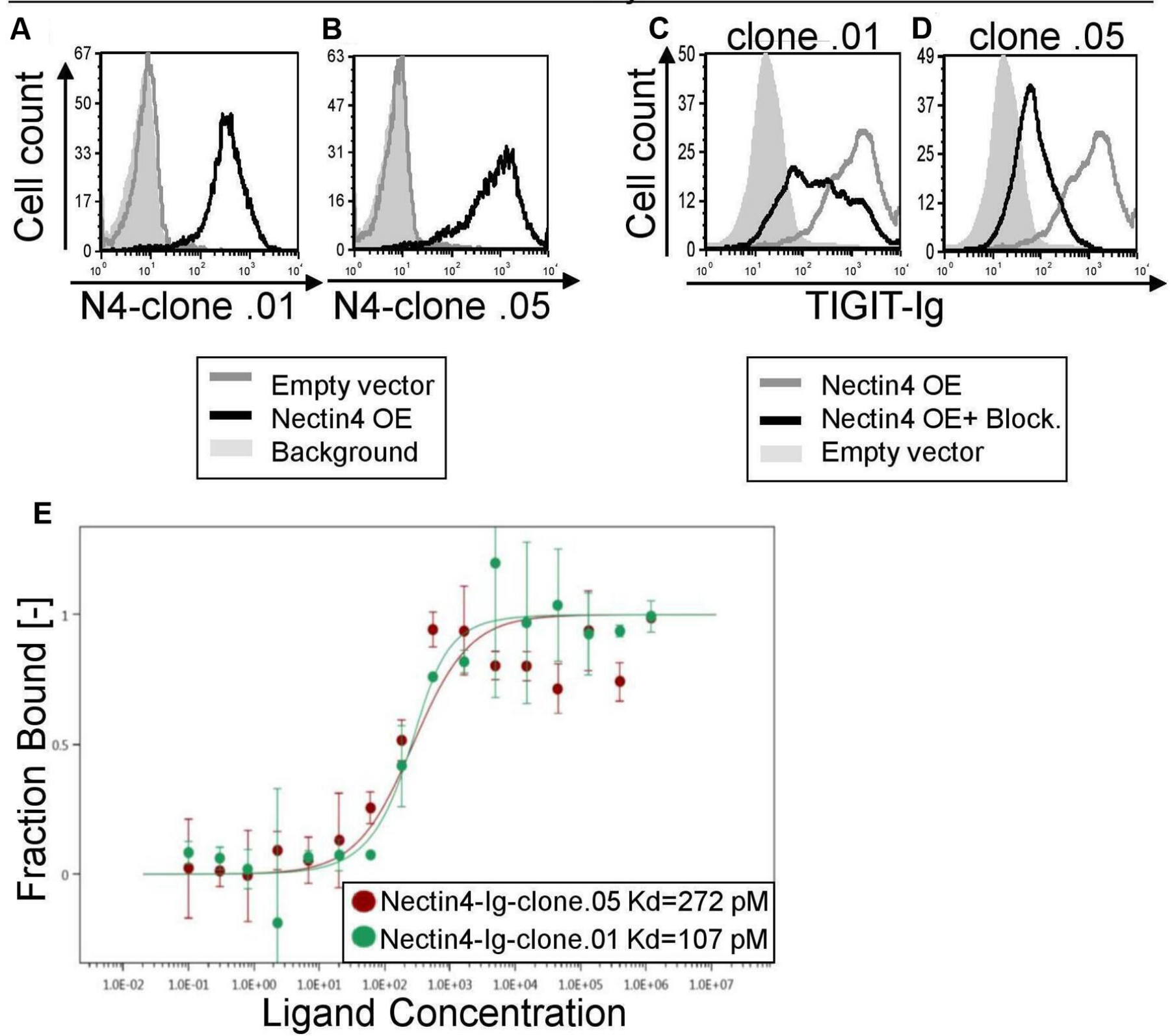

Figure 3 Novel checkpoint inhibitor anti-Nectin4 antibodies block TIGIT binding. (A, B) FACS staining of Raji cells transfected either with an empty vector (gray histograms) or with Nectin4 over-expression (black histograms). Gray filled histograms represent background staining of secondary antibody only. Cells were stained with anti-Nectin 4 mAb clone .01 (A) or clone .05 (B). (C, D) FACS staining with TIGIT-Ig of Raji overexpressing Nectin4 with (black histograms) or without (gray histograms) preincubation with anti-Nectin4 mAb clone .01 (C) or clone .05 (D). Gray, filled histograms represent background staining. Figures show one representative experiment out of three performed. Graph depicting the mean fluorescence intensity values of the stainings appears in online supplementary figure 3a-d. (E) Avidity quantification between Nectin4-Ig and anti-Nectin4 mAb clone .01 and clone .05 using MST. Measurements were repeated with at least three independent protein preparations. FACS, fluorescence-activated cell sorting; MST, microscale thermophoresis

Next, we wanted to assess the mAb's effect in the context of other TIGIT ligands when Nectin4 is expressed endogenously. To this end, we subcutaneously injected MDAMB-453 cells (which express Nectin4 and other ligands from the Nectin family, online supplementary figure 11-n) into SCID-beige mice with or without human NK cells. Mice were treated with clone .05 or a control Ab twice a week, while tumors were visible (indicated by arrows in figures 6,7). As shown, tumor weight was similar in the presence or absence of the antibody, when no NK cells were injected, indicating that clone .05 by itself does not affect tumor growth (figure 7A,B). When NK cells were injected along with clone .05 , however, tumor growth was suppressed (figure 7C,D).

Finally, we show that high expression of Nectin4 is associated with poor overall survival in cancer patients 

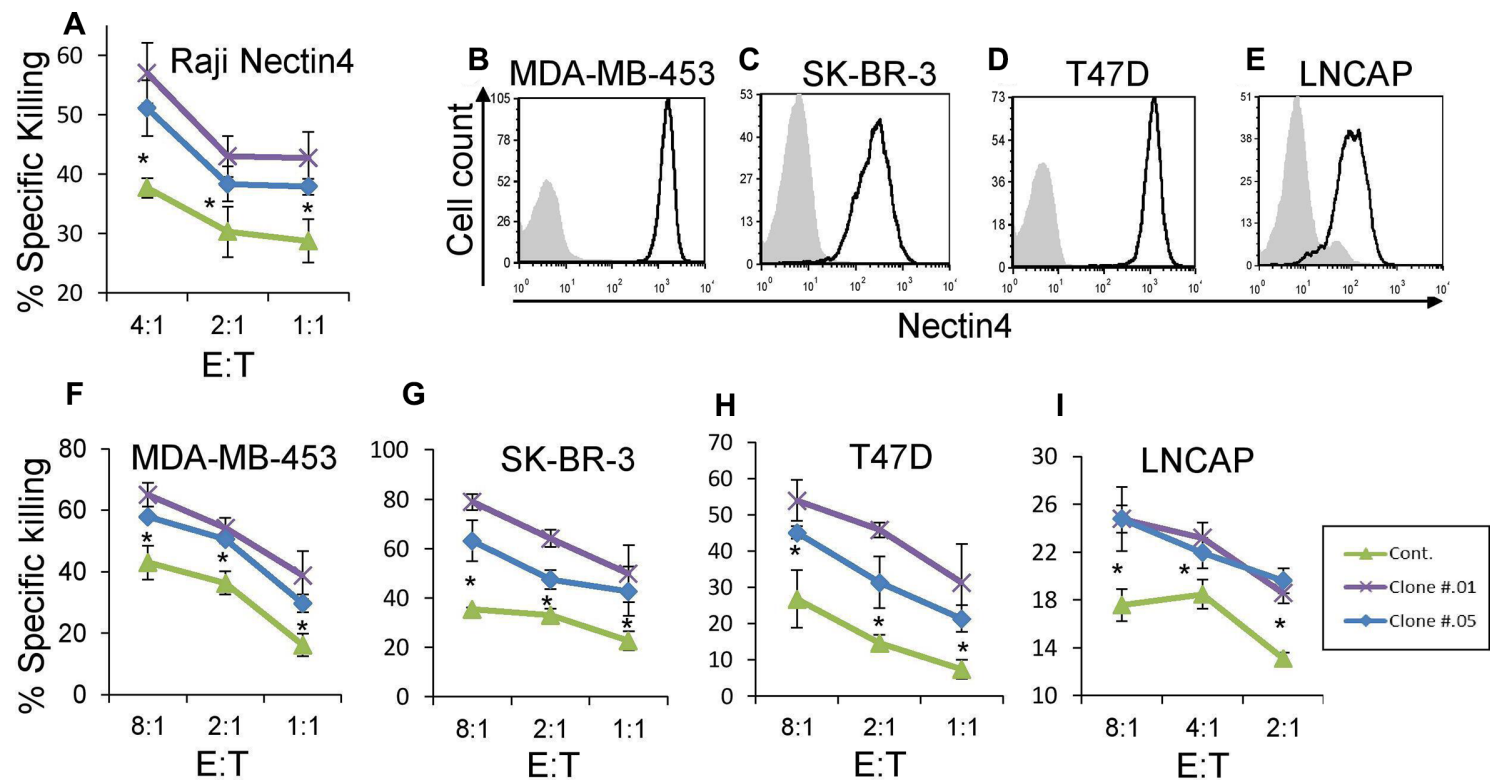

Figure 4 Anti-Nectin4 antibodies increase NK cytotoxicity. (A) $\left[{ }^{35} \mathrm{~S}\right]$ methionine-labeled Raji cells transfected with Nectin4 were incubated with $1 \mu \mathrm{g} / \mathrm{well}$ of either mouse IgG1 as control Ab (green) or with clone .01 (purple) or clone .05 (blue) for 1 hour and then incubated with activated human NK cells for 5 hours. The effector to target (E:T) ratios are indicated on the X-axis. Figure shows one representative experiment out of three performed. Shown is the relative average killing $\pm S D$., ${ }^{*} p<0.05$ (significance between the clones' blocking and control antibody blocking). (B-E) FACS staining of cell lines stained with commercial antiNectin4 antibody (black line histograms). Gray filled histograms are background staining with secondary antibody only. Cell lines used are MDA-MB-453 (B), SK-BR-3 (C), T47D (D) and LNCaP (E). Figures show one representative experiment out of three performed. Graph depicting the mean fluorescence intensity values of the stainings appears in online supplementary figure 3eh. $(F-I)\left[{ }^{35}\right.$ S] methionine-labeled MDA-MB-453 (F), SK-BR-3 (G), T47D (H), and LNCaP (I) cells were incubated with either mouse IgG1 as control mAb (green) or with clone .01 (purple) or clone .05 (blue) for 1 hour and then incubated with activated human NK cell cultures for 5 hours. The E:T ratios are indicated on the X-axis. Figures show one representative experiment out of three performed. Shown is the relative average killing $\pm S D$., " $p<0.05$ (significance between the clones' blocking and the control antibody). FACS, fluorescence-activated cell sorting; NK, natural killer.

(figure 7E) and marks a poorer overall prognosis in several cancer types. ${ }^{18}$ Therefore, further investigation of our antibody as a drug for these patients is of great clinical value.

\section{DISCUSSION}

Checkpoint inhibitors such as anti-CTLA-4 and anti-PD-1 have revolutionized cancer therapy, but they can carry
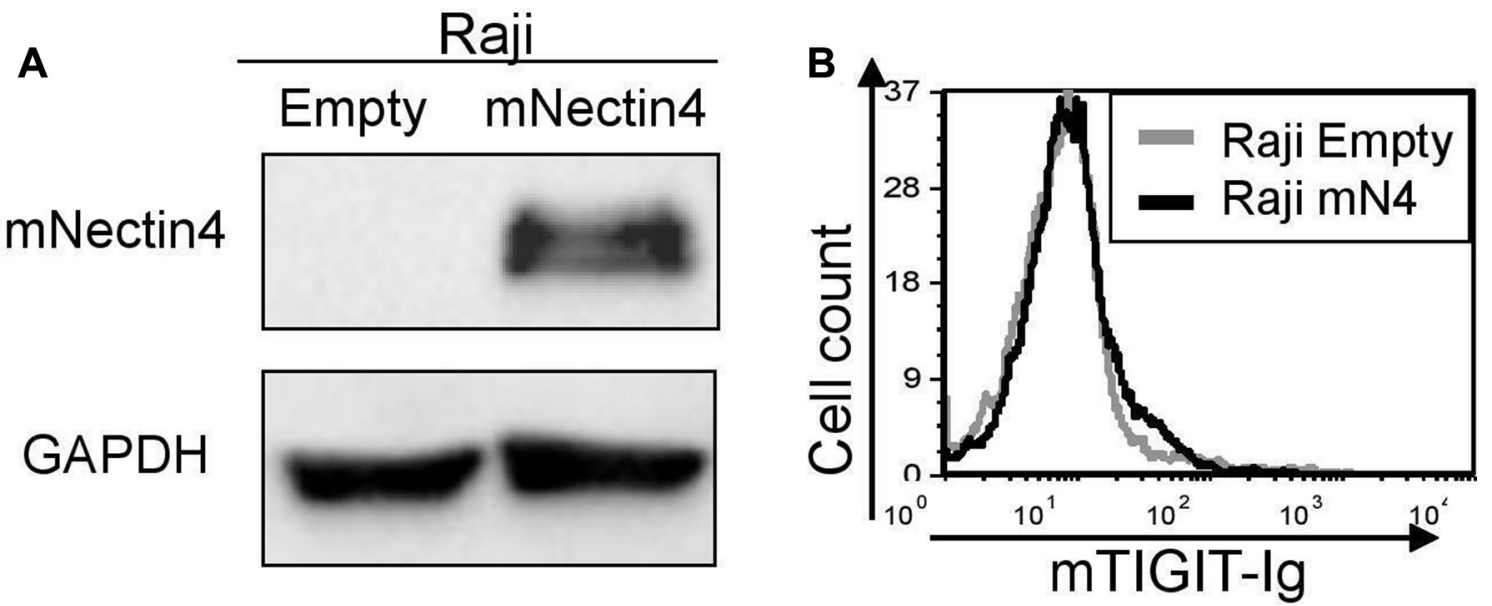

Figure 5 Murine Nectin4 does not bind murine TIGIT. (A) Overexpression of murine Nectin4 (indicated as mNectin4) on Raji cells. Western blots were performed with antimurine Nectin 4 AB and expression was compared with Raji cells expressing empty vector (indicated as empty). Staining for GAPDH was used as a loading control. (B) FACS staining of Raji cells transfected either with an empty vector as control (gray histograms), or with murine Nectin4 (black histograms). Cells were stained with murine TIGIT-Ig. Figures show one representative experiment out of three performed. Graph depicting the mean fluorescence intensity values of the stainings appears in online supplementary figure 4 FACS, fluorescence-activated cell sorting. 


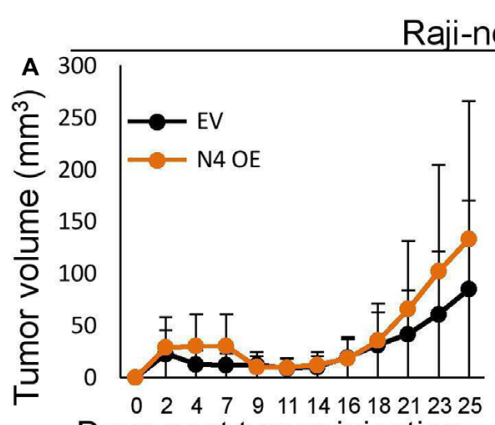

Days post tumor injection

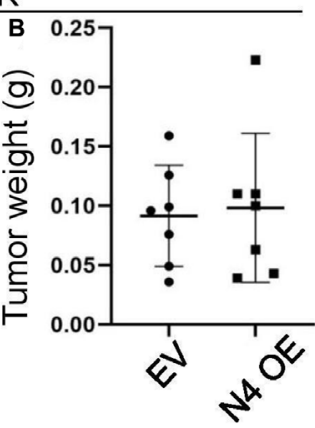

Raii+NK

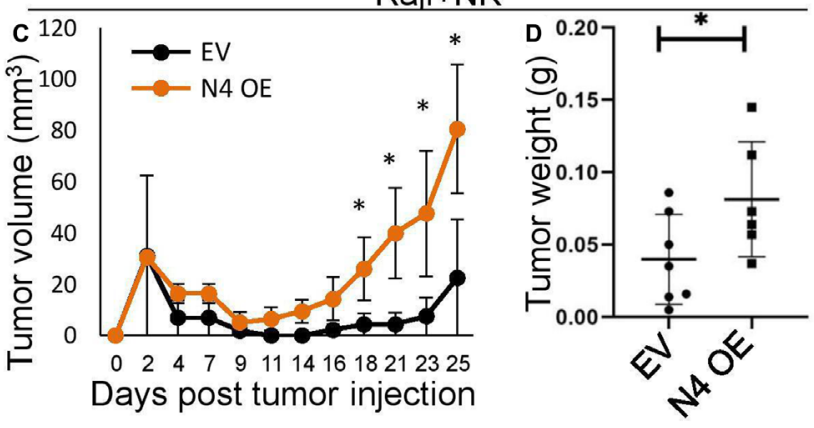

Raji-Nectin4 OE+NK
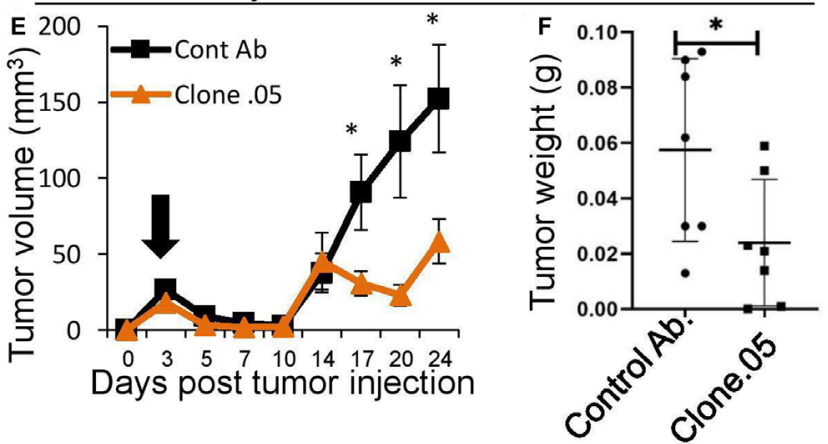

Figure 6 In vivo effect of anti-Nectin4 mAb on tumors overexpressing Nectin4. (A-D) SCID-beige mice were subcutaneously implanted with $5 \times 10^{6}$ Raji cells either overexpressing Nectin4 (N4 oe) or empty vector (EV) either alone $(A, B)$ or with $1 \times 10^{6} \mathrm{NK}$ cells $(C, D)$. (E, F) SCID-beige mice were subcutaneously implanted with $5 \times 10^{6}$ Raji cells overexpressing Nectin4 (N4 oe) and $1 \times 10^{6} \mathrm{NK}$ cells. Mice were then treated with either anti-Nectin 4 clone $.05 \mathrm{mAb}$ (clone .05) and or a control Ab. (A, C, E) Tumor growth was followed with standard caliper. Starting day of antibody treatment is marked by a black arrow. Tumor volumes were calculated by the formula: length $\times$ width ${ }^{2} \times 0.5 .^{21}(B, D, F)$ Tumors ware harvested and weighed on day $21(B, D)$ or 27 (F) post tumor injection. For all murine experiments $n=7$. ${ }^{*} \mathrm{p}<0.05$. NK, natural killer.

many side effects. Much of this is attributed to off-target immune activation. ${ }^{1}$ Anti TIGIT blocking mAbs have also been shown to induce immune-related adverse events. ${ }^{19}$

Here, we report that Nectin 4 is a previously undescribed TIGIT ligand and characterize a Nectin4 blocking antibody we generated. As Nectin4 exhibits tumor specificity, ${ }^{11}$ our antibody aims to induce an immune response specifically against the tumor, thereby minimizing side
MDA-MB-453-no NK
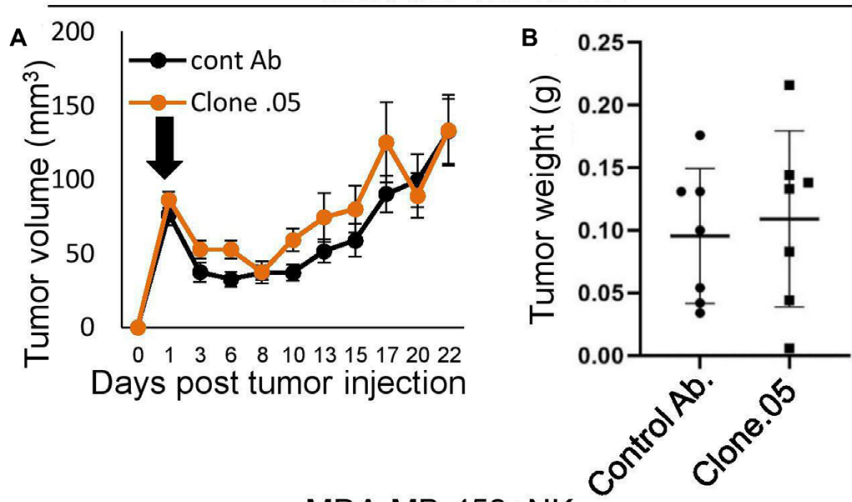

MDA-MB-453+NK
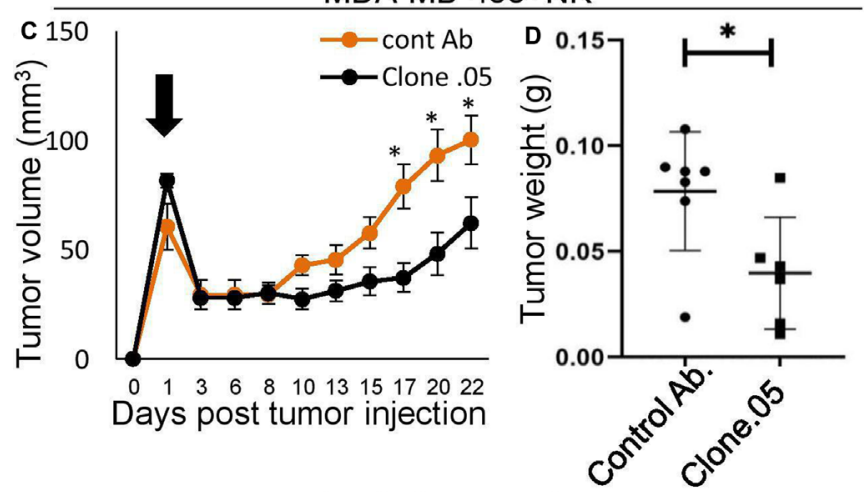

Nectin4 expression
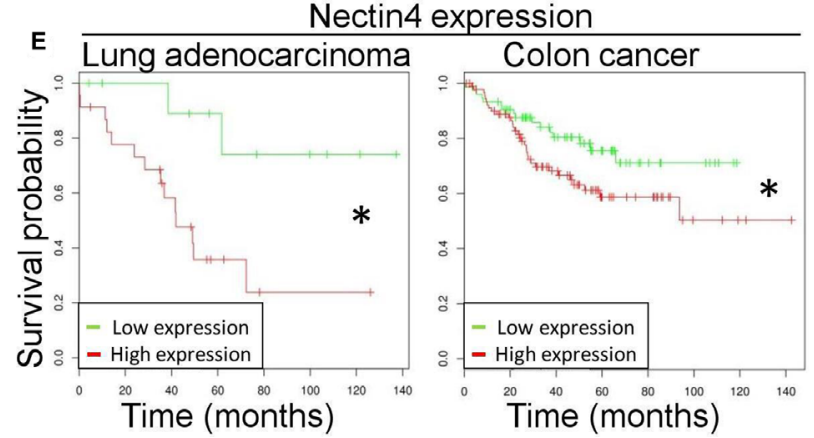

Figure 7 The checkpoint inhibitor anti-Nectin4 mAb enhances NK killing of tumors expressing all ligands of TIGIT in vivo. (A-D) SCID-beige mice were subcutaneously implanted with $5 \times 10^{6} \mathrm{MDA}-\mathrm{MB}-453$ cells either alone (A, B) or with $7 \times 10^{5} \mathrm{NK}$ cells (C, D). Half of each group was treated with anti-Nectin 4 clone $.05 \mathrm{mAb}$ (clone.05) or a control Ab. (A, C) Tumor growth was followed with standard caliper. starting day of antibody treatment is marked by a black arrow. Tumor volumes were calculated by the formula: length $\times$ width $^{2} \times 0.5 .^{21}$ $(B, D)$ Tumors ware harvested and weighed on day 23 posttumor injection. For all murine experiments $n=7 .{ }^{*} P<0.05$. (E) Kaplan-Meier curves of lung adenocarcinoma patients (left, Geo dataset ID: GSE36471) and colon cancer patients (right, Geo dataset ID: GSE17538) after stratification by Nectin4 expression level. ${ }^{*} P<0.05$. Data were obtained from DRUGSURV. ${ }^{22} 23 \mathrm{NK}$, natural killer

effects. This is in contrast to other checkpoint inhibitors, which may have a more general effect on the immune system and lead to autoimmunity. As we have shown, unlike other ligands in its family, Nectin4 binds only to TIGIT. TIGIT is a powerful immune checkpoint as it is expressed on TILs and is associated with immune cell exhaustion. ${ }^{34}$ 
TIGIT binds several ligands, including Nectin1, Nectin2 and Nectin3, in order to allow close monitoring and fine tuning of this immune checkpoint. All known TIGIT ligands are also bound by other immune receptors such as the inhibitory receptors CD112R and CD96 (that sometimes is considered as activating ${ }^{20}$ ) and the coactivating receptor DNAM1, which physically influence ligand availability for TIGIT binding. ${ }^{2}$ It is, therefore, hard to predict how blocking of TIGIT will influence tumor growth as it might affect other receptors in the system. In contrast, here, we show in vitro and in vivo that blocking Nectin4 which, as we show, interacts with TIGIT only, leads to increased tumor killing. Therefore, blocking Nectin4TIGIT interaction using anti-Nectin $4 \mathrm{mAb}$, may lead to significant and specific induction of the immune system against tumors.

The synergy of a purely inhibitory effect, tumorrestricted expression, and its status as an immune checkpoint ligand makes Nectin 4 a unique candidate for cancer immunotherapy. Our blocking antibody is, therefore, the founding member of a new generation of tumor-specific checkpoint inhibitors which can enhance tumor killing in a specific manner with minimal side effects.

\section{CONCLUSIONS}

In this study, we introduce Nectin 4 as a novel major ligand for TIGIT, an inhibitory checkpoint receptor. We further generate anti Nectin 4 antibodies that block its interaction with TIGIT and demonstrate that they increase tumor killing in vitro and in vivo. We expect these new anti Nectin 4 antibodies to become a new form of cancer specific, autoimmune side effect free, cancer checkpoint immunotherapy.

Acknowledgements The authors would like to thank Daphna Burowski for her excellent advice, as well as Karmela Miklic and Suzana Malic for technical support.

Contributors AR conducted experiments, analysed the data and wrote the manuscript. YO initiated the project. IK conducted part of the initial screening. PT and BS advised. BI and YCA contributed to the in vivo studies. NS edited the manuscript. PKB and TL generated blocking antibodies against Nectin4. SJ provided reagents and supervised the project. OM supervised the project and wrote the manuscript.

Funding AR is supported by the Einstein Kaye scholarship. This work was supported by the Israel Innovation Authority (Kamin grant), the Israel Science Foundation (Moked grant), the GIF Foundation, the ICRF professorship grant, the ISF Israel-China grant, and the Ministry of Science and Technology grant (all to 0.M.). SJ was supported by the grant 'Strengthening the capacity of CerVirVac for research in virus immunology and vaccinology', KK.01.1.1.01.0006, awarded to the Scientific Centre of Excellence for Virus Immunology and Vaccines.

Competing interests None declared.

Patient consent for publication Not required.

Ethics approval The research protocol was reviewed and approved by the Ethics Committee of the Hebrew University (Ethical approval number: MD-17-15165-4).

Provenance and peer review Not commissioned; externally peer reviewed.

Data availability statement Data are available on reasonable request. All data relevant to the study are included in the article or uploaded as online supplementary information. Data are available in a public, open access repository.

Open access This is an open access article distributed in accordance with the Creative Commons Attribution Non Commercial (CC BY-NC 4.0) license, which permits others to distribute, remix, adapt, build upon this work non-commercially, and license their derivative works on different terms, provided the original work is properly cited, appropriate credit is given, any changes made indicated, and the use is non-commercial. See http://creativecommons.org/licenses/by-nc/4.0/.

\section{ORCID iD}

Ofer Mandelboim http://orcid.org/0000-0002-9354-1855

\section{REFERENCES}

1 Wang DY, Salem J-E, Cohen JV, et al. Fatal toxic effects associated with immune checkpoint inhibitors. JAMA Oncol 2018;4:1721.

2 Stein N, Tsukerman P, Mandelboim O. The paired receptors TIGIT and DNAM-1 as targets for therapeutic antibodies. Hum Antibodies 2017;25:111-9.

3 Josefsson SE, Huse K, Kolstad A, et al. T cells expressing checkpoint receptor TIGIT are enriched in follicular lymphoma tumors and characterized by reversible suppression of T-cell receptor signaling. Clin Cancer Res 2018;24:870-81.

4 Yu X, Harden K, C Gonzalez L, et al. The surface protein TIGIT suppresses $\mathrm{T}$ cell activation by promoting the generation of mature immunoregulatory dendritic cells. Nat Immunol 2009;10:48-57.

5 Kučan Brlić P, Lenac Roviš T, Cinamon G, et al. Targeting Pvr (CD155) and its receptors in anti-tumor therapy. Cell Mol Immunol 2019;16:40-52.

6 Miao X, Yang Z-L, Xiong L, et al. Nectin-2 and DDX3 are biomarkers for metastasis and poor prognosis of squamous cell/ adenosquamous carcinomas and adenocarcinoma of gallbladder. Int $J$ Clin Exp Pathol 2013;6:179-90.

7 Mizutani K, Takai Y. Nectin spot: a novel type of nectin-mediated cell adhesion apparatus. Biochem J 2016;473:2691-715.

$8 \mathrm{Xu} \mathrm{F,} \mathrm{Si} \mathrm{X,} \mathrm{Wang} \mathrm{J,} \mathrm{et} \mathrm{al.} \mathrm{Nectin-3} \mathrm{is} \mathrm{a} \mathrm{new} \mathrm{biomarker} \mathrm{that} \mathrm{mediates}$ the upregulation of MMP2 and MMP9 in ovarian cancer cells. Biomed Pharmacother 2019;110:139-44.

9 Zhu Y, Paniccia A, Schulick AC, et al. Identification of CD112R as a novel checkpoint for human T cells. J Exp Med 2016;213:167-76.

10 M-Rabet M, Cabaud O, Josselin E, et al. Nectin-4: a new prognostic biomarker for efficient therapeutic targeting of primary and metastatic triple-negative breast cancer. Ann Oncol 2017;28:769-76.

11 Challita-Eid PM, Satpayev D, Yang P, et al. Enfortumab Vedotin antibody-drug conjugate targeting Nectin-4 is a highly potent therapeutic agent in multiple preclinical cancer models. Cancer Res 2016;76:3003-13.

12 Ophir Y, Duev-Cohen A, Yamin R, et al. Pilra binds an unknown receptor expressed primarily on CD56bright and decidual-NK cells and activates NK cell functions. Oncotarget 2016;7:40953-64.

13 Dassa L, Seidel E, Oiknine-Djian E, et al. The human cytomegalovirus protein UL148A downregulates the NK cell-activating ligand MICA to avoid NK cell attack. J Virol 2018;92:e00162-18.

14 Stanietsky N, Simic H, Arapovic J, et al. The interaction of TIGIT with Pvr and PVRL2 inhibits human NK cell cytotoxicity. Proc Natl Acad Sci U S A 2009;106:17858-63.

15 Elias S, Kahlon S, Duev-Cohen A, et al. A BW reporter system for studying receptor-ligand interactions. J Vis Exp 2019;2019:1-13.

16 Berhani O, Glasner A, Kahlon S, et al. Human anti-NKp46 antibody for studies of NKp46-dependent NK cell function and its applications for type 1 diabetes and cancer research. Eur J Immunol 2019;49:228-41.

17 Seidel E, Le VTK, Bar-On Y, et al. Dynamic co-evolution of host and pathogen: HCMV downregulates the prevalent allele MICA $* 008$ to escape elimination by NK cells. Cell Rep 2015;10:968-82.

18 Zhang Y, Zhang J, Shen Q, et al. High expression of Nectin-4 is associated with unfavorable prognosis in gastric cancer. Oncol Lett 2018;15:8789-8795-95.

19 OncoMed Announces Early Clinical Data For Anti-TIGIT Antibody [Internet]. Available: https://www.clinicalleader.com/doc/oncomedannounces-early-clinical-data-for-anti-tigit-antibody-0001 [Accessed cited 2020 Mar 3].

20 Georgiev H, Ravens I, Papadogianni G, et al. Coming of age: CD96 emerges as modulator of immune responses. Front Immunol 2018;9:1-10.

21 Tomayko MM, Reynolds CP. Determination of subcutaneous tumor size in athymic (nude) mice. Cancer Chemother Pharmacol 1989;24:148-54.

22 Amelio I, Gostev M, Knight RA, et al. DRUGSURV: a resource for repositioning of Approved and experimental drugs in oncology based on patient survival information. Cell Death Dis 2014;5:e1051.

23 Antonov AV. BioProfiling.de: analytical web portal for high-throughput cell biology. Nucleic Acids Res 2011;39:W323-7. 